\title{
Uterine Contraction Stress Test
}

National Cancer Institute

\section{Source}

National Cancer Institute. Uterine Contraction Stress Test. NCI Thesaurus. Code C92908.

A test done to check for the fetal well being after administering oxytocin or nipple stimulus to induce uterine contractions in the mother. An external fetal heart rate monitor is used to assess the fetal heart rate in response to the uterine contractions. 